\section{Estudo \\ CoDebate}

em Cestão

Plamejamento

\title{
OFERTA DE TRABALHO DA TERCEIRA IDADE: ANÁLISE DO PERFIL DO IDOSO INSERIDO NO MERCADO DE TRABALHO BRASILEIRO
}

\author{
Samanda Silva Da Rosa ${ }^{1}$, Gibran Da Silva Teixeira ${ }^{2}$, Márcio Nora Barbosa ${ }^{3}$
}

\begin{abstract}
Resumo: $\mathrm{O}$ presente artigo tem como finalidade traçar um perfil do idoso inserido no mercado de trabalho Brasileiro. Para a realizaçáo deste objetivo, utilizou-se o modelo econométrico de resposta qualitativa logit, para a obtenção das razóes de chances dos idosos brasileiros estarem inseridos no mercado de trabalho, a partir de variáveis independentes selecionadas. A amostra foi construída a partir de dados fornecidos pela Pesquisa Nacional de Amostra por Domicílios, a PNAD, para os anos de 2004 e 2014. Os resultados dos modelos apresentaram razóes de chances maiores para um perfil de idoso inserido no mercado de trabalho brasileiro como sendo do sexo masculino, aposentado e inserido principalmente no comércio. Assim como através de cenários elaborados nota-se a renda como fator preponderante para ambos os sexos se manterem no mercado de trabalho.
\end{abstract}

Palavras-chave: Idoso. Mercado de trabalho. Brasil.

1 Atualmente é mestranda em Economia Aplicada pela Universidade Federal do Rio Grande (2016), possui graduação em Engenharia Agrícola pela Universidade Federal de Pelotas (2010) e graduação em Ciências Econômicas pela Universidade Federal do Rio Grande (2015).

2 Possui graduação em Ciências Econômicas pela Universidade Federal do Rio Grande (2004), mestrado em Economia pela Universidade Federal da Paraíba (2009) e doutorado em Programa de Pós-Graduaçáo em Economia pela Universidade Federal do Rio Grande do Sul (2013). Atualmente é professor Adjunto da Universidade Federal do Rio Grande e professor do Programa de Pós-Graduação em Economia Aplicada - PPGE/Mar/FURG. Tem experiência na área de Economia atuando principalmente nos seguintes temas: Economia do Trabalho, Avaliação de Política Pública e Métodos Quantitativos.

3 É mestre em Economia Aplicada, pelo Programa de Pós-graduação em Economia Aplicada _ PPGE/FURG e especialista em Comércio Exterior e Negociação Internacional pela FGV-Florianópolis, atua como Professor temporário no curso de Comércio Exterior na FURG-SVP, assim como atua na Unidade de Pesquisa em Economia Costeira - UPEC/FURG, onde participa de projetos de pesquisa na área econômica. Possui graduaçáo em Bacharelado em Ciências Econômicas pela Universidade Federal do Rio Grande (2006) e técnico em Comércio Exterior. Tem experiência na área de análise econômica em cadeias produtivas e análise de custo de processos, Logística de commodities e Logística interna. 


\title{
LABOR SUPLY FOR THE ELDERLY: ANALYSIS OF ELDERLY PROFILE INSERTED IN THE BRAZILIAN LABOR MARKET
}

\begin{abstract}
The aim of this paper is to reach a profile of the elderly that are inserted in the Brazilian labor market. The logit econometric model of qualitative response, using some independent variables, was used to obtain the probability of the Brazilian elderly to be included in the labor market. The sample was constructed from data provided by the National Household Sample Survey (PNAD) from 2004 to 2014. The results showed that the elderly profile inserted in the labor market is a person of the male sex, retired and inserted mainly in the commerce. Besides, some scenarios were elaborated, and the income was seen as a preponderant factor for both sexes (male and female) to enter in the labor market.
\end{abstract}

Keywords: Elderly. Labor market. Brazil.

Classificaçáo JEL: J31, J14.

\section{INTRODUÇÁO}

O presente artigo tem como principal objetivo identificar o perfil do trabalhador idoso inserido no mercado de trabalho brasileiro com base nos anos de 2004 e 2014. Para tanto, utilizou-se a base de dados da Pesquisa Nacional por Amostra de Domicílios, PNAD, dos anos de 2004 e 2014. A partir dos resultados, será possível entender a alocação dos idosos abordados, e observar as alteraçóes no decorrer de uma década, assim como será possível visualizar as mudanças de probabilidade de inserção dos idosos com aplicação de cenários específicos.

Nos últimos anos, o fenômeno da longevidade da população mundial, está diretamente associado de maneira positiva, devido os avanços da medicina e da tecnologia. Segundo Leeson e Harper (2008) a população de idosos em todo o mundo passou de 205 milhóes (8\% da população total) em 1950, para cerca de 688 milhóes (11\% da população total) em 2006. No Brasil, o total de idosos ultrapassou os $10 \%$ da população em 2007, contra $8 \%$ em 1995, em poucas décadas, o país passou a enfrentar o desafio do envelhecimento de sua população. De forma que, condiçóes mais favoráveis de saúde proporcionam o aumento da expectativa de vida e, assim sendo, a expansão da população idosa (QUEIROZ; RAMALHO, 2009).

$\mathrm{O}$ oposto acontece com a taxa de natalidade, que vem retraindo-se com o passar do tempo, o que ajuda a transformar a configuração da pirâmide etária brasileira (Organização das Naçóes Unidas, 2009). A previsão para o ano de 2020 é de que o país possua $15 \%$ da população com idade acima de 60 anos, haja vista a influência de fatores relacionados à alta expectativa de vida e à baixa taxa de natalidade (CAMARANO, 2002).

Como no Brasil, a idade em que os idosos saem do mercado de trabalho é elevada, um percentual maior dessa parcela da população, repercute, também em um aumento do número de pessoas acima dos 60 anos inseridas no mercado de trabalho. Em 1997, a parcela de $25 \%$ da população idosa estava em atividade, à tendência para 2020 é que esse número passe para $13 \%$. Ou seja, um percentual menor da população idosa estará em atividade, porém, compensada pelo maior número de idosos no país (WAJNMAN et al., 1999). 
Uma das consequências do envelhecimento populacional é o alto custo para os cofres públicos para o pagamento das pensóes e a sua sustentabilidade, sobretudo, devido aos trabalhadores do mercado informal, onde não há contribuição para a Previdência. Para Oliveira et al. (1997), o número de pessoas seguradas pela previdência social era baixo até meados da década de 70 , no entanto, houve uma elevação considerável nas últimas décadas, no ano de 1994 chegou a cerca de 15 milhóes de beneficiários. Além disso, há as aposentadorias especiais, onde muitas pessoas têm o direito de se aposentar mais cedo, o que faz com que passem um período maior de tempo recebendo o benefício da aposentadoria (FRANÇA, 2012).

Segundo Queiroz e Ramalho (2009), a elevação da participação de idosos no mercado de trabalho, inclusive dos aposentados, pode ser indício da necessidade da mudança no padrão de vida, onde um complemento na renda pode gerar melhora nas condiçóes mínimas de sobrevivência. A expansão de uma parcela maior de idosos na população é refletida no mercado de trabalho, onde, a participação dos trabalhadores mais velhos na dinâmica ocupacional, gera novas dimensóes e configuraçóes no cenário do mercado de trabalho (CAMARANO, 2001; FRANÇA, 2012; GIATTI; BARRETO, 2003).

Diversas organizaçóes estimulam, por um lado, a permanência de profissionais mais velhos por serem altamente especializados, haja vista, de já possuir a aptidão necessária para desempenhar determinada tarefa, tendo em vista sua experiência e conhecimento acumulado; de outro, uma parcela considerável dos próprios trabalhadores, mesmo após atingir uma determinada idade, considerada avançada, almejam continuar colaborando com sua força de trabalho, desde que contem com as suas funções psíquicas e físicas em bom estado. No entanto, há trabalhadores que desejam aposentar-se de modo a não ofertar mais sua força de trabalho, aspirando passar mais tempo com seus familiares, realizando atividades de lazer, ou ainda, realizando outros sonhos (FRANÇA et al., 2013).

Assim, o presente estudo visa contribuir com a literatura sobre a inserçáo do idoso no mercado de trabalho brasileiro, avaliando principalmente o diferencial passado a década de 2004 a 2014. Em geral, os resultados encontrados na avaliação empírica deste estudo mostram que idosos do sexo masculino, aposentados e que trabalham no comércio têm mais chances de estarem inseridos no mercado de trabalho. A renda e o número de componentes da família também se destacam na pesquisa.

Dado o exposto acima, o artigo encontra-se estruturado em mais cinco seçôes, além desta introdução. $\mathrm{Na}$ seção dois realizam-se as evidências para a participação do idoso no mercado de trabalho. Na terceira seção explicitam-se os procedimentos metodológicos e a natureza dos dados que serão utilizados nas estimações. $\mathrm{Na}$ seção quatro são apresentados e discutidos os resultados obtidos dos modelos estimados, assim como a elaboração de cenários específicos para análise de impactos nas probabilidades dos idosos se inserirem no mercado de trabalho. Por fim, a quinta seção, traz as consideraçóes finais. 


\section{EVIDÊNCIAS SOBRE A PARTICIPAÇÁO DOS IDOSOS NO MERCADO DE TRABALHO}

\subsection{A População Idosa no Brasil}

Nas próximas décadas, o mundo terá uma população de idade cada vez mais elevada, as projeçóes são de que em 2050, a população de idosos (com idade a partir de 60 anos) tenha um aumento de $20 \%$ para um nível superior a $30 \%$ nos países mais desenvolvidos, de $8 \%$ para $20 \%$ em países menos desenvolvidos (LEESON; HARPER, 2008). No Brasil, a expectativa de sobrevida do homem idoso teve uma elevação de $54 \%$ e a mulher idosa teve a expectativa de sobrevida elevada em 64\% entre os anos de 1930 e 2009. As taxas de fertilidade no Brasil estão próximas das registradas em países desenvolvidos, saindo de 6,3 em 1960 para 1,94 em 2009. A combinação destes fatores tem corroborado para a queda da taxa média anual de crescimento da população (MINISTÉRIO DA PREVIDÊNCIA SOCIAL, 2011).

A Figura 1, a seguir, mostra a distribuição da população por gênero a partir do Instituto Brasileiro de Geografia e Estatística (IBGE), segundo os grupos de idade no Brasil nos anos de 2000 e 2010 . Os dados indicam que no período observado houve um estreitamento da base e alargamento do seu topo da pirâmide. $\mathrm{O}$ total de idosos com 60 anos de idade ou mais cresceu em torno de $41 \%$ entre 2000 e 2010 . Já o total de crianças e jovens de 0 a 19 anos diminuiu aproximadamente $8 \%$ no mesmo período. É possível destacar o predomínio das mulheres na população idosa. O total de mulheres idosas é $25 \%$ maior ao total de homens idosos no ano de 2010, a mesma taxa em 2000 era de 23\%. Essa diferença deve-se à maior expectativa de vida atribuída às mulheres.

Figura 1: Brasil - Distribuição da população por faixa etária e gênero (2000 e 2010)

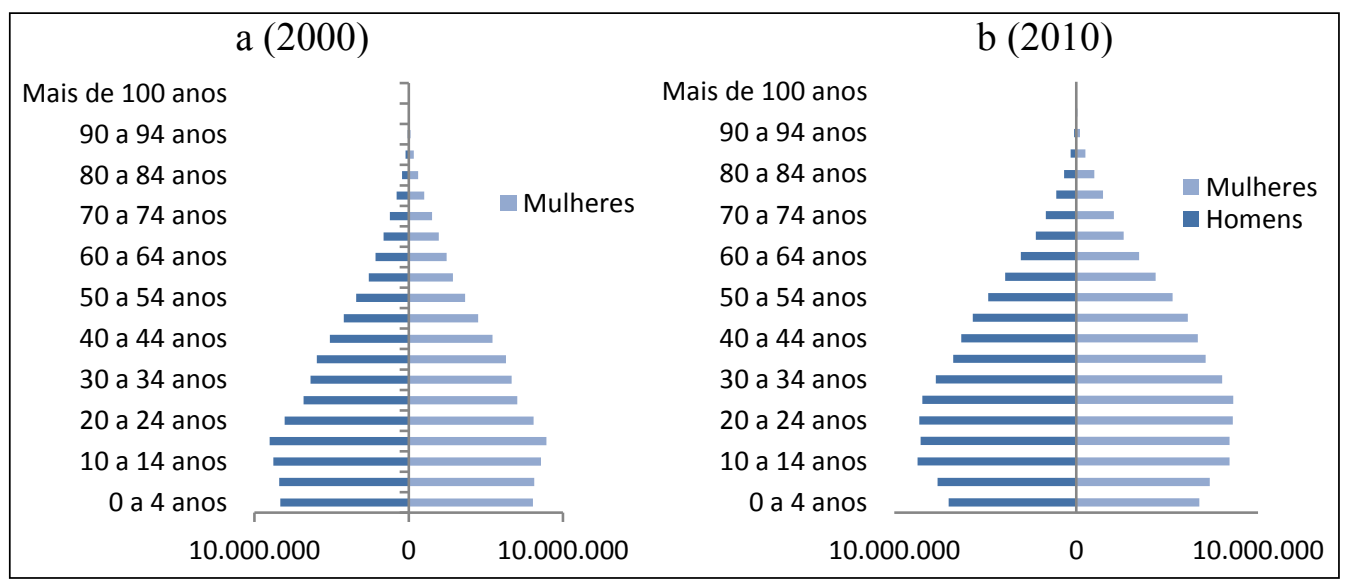

Fonte: Elaborado pelos autores com base nos dados dos Censos Demográficos de 2000 e de 2010.

Em linhas gerais, o país está em plena transição demográfica, onde as taxas de mortalidade e fecundidade estão diminuindo e a expectativa de vida aumentando. Vale 
destacar que em alguns países da Europa transição mudança demorou um período superior a 100 anos (CAMARANO et al., 2004).

\subsection{O Idoso no mercado de trabalho}

A seguir são apresentadas relevantes contribuiçóes da literatura em relação à inserção ocupacional dos idosos, começando pela literatura nacional e por seguinte, a literatura internacional.

Em relação aos idosos, a ampliação ou reformas dos programas de Seguridade Social apresentam impacto crucial na escolha de atividade ou inatividade do idoso (GRUBER; WISE, 2004). Segundo Queiroz e Ramalho (2009), a promulgação da Constituição brasileira de 1988, que trouxe benefícios aos seus beneficiários, acarretou em reformas da Previdência em anos posteriores por não conseguir suprir as novas responsabilidades. Para Furtado (2005), um percentual de 46\% dos idosos do sexo masculino no ano de 2003 , participava ativamente no mercado de trabalho, superando índices de países desenvolvidos.

De acordo com Leme e Málaga (2001), o idoso que recebe aposentadoria, que representa uma garantia, considera alguns empregos disponíveis menos atraentes, principalmente considerando um nível de instrução mais elevado. Para Liberato (2003), continuar trabalhando após a aposentadoria é considerada uma forma de compensar a perda do poder aquisitivo, principalmente para os idosos que têm um nível de escolaridade mais elevado. No Brasil, a atividade de trabalho aliada a aposentadoria podem ser um importante instrumento para o combate à pobreza familiar e estimular a atividade do idoso (CAMARANO, 2001; WAJANMAN et al., 2004).

Segundo, Carrera-Fernandes e Menezes (2001), na região metropolitana de Salvador a hipótese de que o idoso retorna ao mercado de trabalho como forma de terapia ocupacional é rejeitada. A decisão de continuar no mercado de trabalho deve-se aos ganhos salariais obtidos. Logo, a aposentadoria, pensão e seguro desemprego são indicadores cruciais na tomada de decisão da continuidade da oferta de trabalho.

Na cidade de Sáo Paulo, o estudo sobre a participação dos idosos no mercado de trabalho teve, como enfoque principal a questão da saúde como condição para continuar ofertando mão de obra após a aposentadoria. De acordo com a pesquisa, uma situação desfavorável de saúde, promove menor probabilidade de continuar ativou ou de trabalhar um maior número de horas. No momento em que, a variável saúde é levada em consideração, a idade e a escolaridade perdem parte do seu poder explicativo (PÉREZ; WAJNMAN; OLIVEIRA, 2006).

Segundo Normanha Filho (2004), o trabalhador idoso tem seu conhecimento construído através de vários fatores, entre eles o período trabalhou, a sua escolaridade e conhecimentos adquiridos de forma independente, a cultura e o local onde viveram. Após aposentado, a renda do idoso não depende mais da sua participação no mercado de trabalho e, por isso, não depende do seu estado de saúde. Para Bós e Bós (2004), a grande maioria dos idosos do Rio Grande do Sul não participa do mercado de trabalho. Eles têm asseguradas outras fontes de renda independentes de atividades de trabalho, principalmente aposentadoria e pensão. Remuneração pelo trabalho é mais comum para idosos mais jovens 
(60 a 64 anos de idade), tornando-se praticamente inexistente para aqueles acima de setenta anos.

No caso do Brasil, Saad (1999), realizou uma pesquisa sobre as "transferências intergeracionais" no âmbito familiar. Relatou que devido à situação econômica do país, muitos filhos adultos continuam a residir com os pais e se mantendo dependentes financeiramente deles. E que, o fato de seus pais receberem aposentadoria e/ou continuar trabalhando ajuda nessa condição.

Para Moura e Cunha (2010), mesmo que o coeficiente de participação do idoso no mercado de trabalho venha caindo com o passar dos anos, os idosos ainda têm uma participação positiva e estatisticamente significativa no mercado de trabalho. Analisando que o rendimento através de salário é o fator que contribui de forma importante para a composição da renda familiar, porém, os idosos possuem fragilidades de inserção no mercado de trabalho, o que demandaria políticas públicas específicas para ajudar esses grupos, tanto em termos de rendimento como nas condiçóes de trabalho.

Ao abordar os determinantes da participação do idoso no mercado de trabalho e dos seus salários no Brasil, Queiroz, Ramalho e Cavalcanti (2005), concluíram que idosos não aposentados apresentam rendimentos mais elevados em comparação aos idosos aposentados, isso, devido ao fato de possuir um grau mais elevado de instrução. E que os fatores determinantes para o idoso permanecer no mercado de trabalho são, o sexo, a posição na família, a localização setorial, o capital humano e rendas não oriundas do trabalho.

De acordo com Gasparini et al. (2007), nos países desenvolvidos, a combinação de sistemas de segurança social, e pequenas famílias contribuem para os padróes de vida mais elevados para os idosos, em relação ao restante da população. Estas condiçóes não são replicadas em muitos países em desenvolvimento, onde os sistemas de pensões são fracos e principalmente desfavorecem os mais pobres, e os idosos geralmente vivem em grandes famílias que compartilham o orçamento com um grande número de pessoas. Seu estudo avalia a situação dos idosos em termos de pobreza, de renda e outras dimensôes do bem-estar na América Latina e Caribe. As pensóes ou quaisquer outros mecanismos de transferência de renda para os idosos são essenciais para manter a velhice abaixo da linha da pobreza (GASPARINI et al., 2007).

\section{METODOLOGIA}

O modelo empírico a seguir procura explicar quais os determinantes do emprego do idoso no mercado de trabalho no Brasil, a partir dos dados das PNADs de 2004 e 2014. Para tanto, será utilizado o modelo econométrico logit.

\subsection{Modelo logit}

A partir dos dados coletados, a metodologia utilizada será o modelo logit. Para Nasir (2005), a utilização do modelo logit se aplica para esta finalidade de pesquisa, conforme a diversificada gama de estudos empíricos sobre o mercado de trabalho do idoso. 
Segundo Queiroz e Ramalho (2009), um problema que deve ser considerado nesse tipo de trabalho é sobre o viés de seleção da amostra. Pois, algum dos grupos pode apresentar alguma característica produtiva que náo foi incluída no estudo, tais como: liderança, entusiasmo, entre outras (HECKMAN, 1979).

Segundo Teixeira (2009), "o modelo econométrico é chamado de modelo linear de probabilidade" no momento em que o valor da variável dependente depende das variáveis explanatórias sendo interpretado como a probabilidade do evento ocorrer. No caso em questão, significa a probabilidade do indivíduo após receber a aposentadoria continuar no mercado de trabalho.

O modelo logit é baseado na função de probabilidade logística acumulada, a qual é representada como:

$$
P i=f\left(Z_{i}\right)=f\left(\propto+\sum_{j} \beta_{j} X_{i j}\right)=\frac{1}{1+e^{-Z i}}=\frac{1}{1+e^{-\left\{\alpha+\sum_{j} \beta_{j} X_{i j}\right\}}}
$$

$\mathrm{O}$ próximo registro será da probabilidade de ocorrer um evento, de acordo com $\mathrm{X}$ observaçóes $\mathrm{i}$, para $1 \leq \mathrm{i} \leq \mathrm{k}$, onde $\mathrm{k}$ é o número de observaçóes. O valor de $\beta_{j}$ é o coeficiente da variável independente, $X_{j}$ e $Z_{j}$ são índices contínuos teóricos determinados pelas variáveis explicativas $X_{j}$, de modo que:

$$
Z_{i}=\sum_{j} \beta_{j} X_{i j}
$$

$\mathrm{O}$ tratamento algébrico sobre a equação conduz a:

$$
L N(P i / 1-P i)=Z_{i}=\propto+\sum_{j} \beta_{j} X_{i j}
$$

De acordo com Teixeira (2009), a variável dependente da equação de regressão é relacionada com a probabilidade de ocorrer um dos dois possíveis eventos, 1 para aposentado e empregado e zero para os indivíduos que são apenas aposentados. Um argumento a favor do modelo logit é a transformação do problema de predição probabilística de um intervalo [0;1] em um problema de predição de probabilidade de ocorrência de eventos no campo da reta real (PINDYCK; RUBINFELD, 1998).

Para estimar os coeficientes do modelo logit será utilizado o método da máxima verossimilhança. Segundo Greene (2003), esse método é recomendado quando a análise é sobre a ocorrência ou não de determinada situação.

$$
P i=1 / 1+e^{-\left(\alpha+\sum_{j} \beta_{j} X_{i j}\right)}
$$

O valor de $P_{i}$ não é individualmente analisado, pois, há apenas a informação de ocorrência ou não de trabalho após a aposentadoria. A variável dependente $Y_{i}$ tem o valor 1 quando ocorre o evento e 0 caso contrário. Logo, para estimar os parâmetros do modelo $(\alpha$ e cada um dos $\beta_{j}$ ) de modo que maximize a probabilidade que o evento da amostra avaliada ocorra, no caso, estar aposentado e empregado.

Para determinar a função de verossimilhança, devem-se relacionar as primeiras observaçóes em que ocorreu o evento $\left(n_{1}\right)$ e as últimas observaçóes em que o evento ocorreu $\left(n_{2}\right)$. De modo que $n_{1}+n_{2}=\mathrm{N}$, onde $\mathrm{N}$ é o tamanho total da amostra.

$$
L=P\left(Y_{1}, Y_{2}, \ldots, Y_{N}\right)=P\left(Y_{1}\right) \cdot P\left(Y_{2}\right) \ldots P\left(Y_{N}\right)
$$


Analisando a probabilidade de não ocorrência do fato ser igual a 1 menos a probabilidade de ocorrer o evento e utilizando-se $\Pi$ para o produto dos fatores, tem-se que:

$$
\begin{aligned}
& L=P_{1} \cdot P_{2} \ldots P_{n 1} \cdot\left(1-P_{n 1+1}\right) \cdot\left(1-P_{n 1+2}\right) \ldots\left(1-P_{N}\right) \\
& L=\prod_{i=1}^{n 1} P_{i} \prod_{i=n 1+1}\left(1=P_{i}\right)
\end{aligned}
$$

Tornando o logaritmo de L:

$$
\log L=\sum_{i=1}^{n 1} \log P_{i}+\sum_{i=n 1+1} \log \left(1-P_{i}\right)
$$

Para calcular as estimativas dos parâmetros, deve-se derivar Log L em função de $\alpha$ e $\operatorname{dos} \beta_{j}$, igualando o resultado a zero, i.e:

$$
\begin{gathered}
\frac{\partial(\log L)}{\partial \propto}=\sum_{i=1}^{n 1} \frac{\partial P_{i} / \partial \propto}{P_{i}}-\sum_{1-P_{i}}^{N} \frac{\partial P_{i} / \partial \propto}{1-P_{i}}=0 \\
\partial P_{i} / \partial \beta_{j} \quad \frac{\partial P_{i} / \partial \beta_{j}}{P_{i}}-\sum_{i=n 1+1}^{N} \frac{}{1-P_{i}}=0 \quad j=1, \ldots, k
\end{gathered}
$$

Sendo:

$$
1 P_{i}=\frac{1}{1+e^{-\left\{\alpha+\Sigma_{j} \beta_{j} X_{j i}\right\}}}
$$

Para obter as estimativas dos coeficientes do modelo logit resolve-se o sistema:

$$
\begin{aligned}
& \sum_{i=1}^{n_{1}} \frac{\left[e^{-\left(\alpha+\sum \beta_{j} X_{j}\right)}\right]}{\left[1+e^{-\left(\alpha+\sum \beta_{j} X_{j}\right)}\right]}-\sum_{i=n_{1}+1}^{N} \frac{\left[e^{-\left(\alpha+\sum \beta_{j} X_{j}\right)}\right] \cdot\left[1+e^{+\left(\alpha+\beta_{j} X_{j}\right)}\right]}{\left[1+e^{-\left(\alpha+\sum \beta_{j} X_{j}\right)}\right]^{2}}=0 \\
& \sum_{i=1}^{n_{1}} \frac{X_{j} \cdot\left[e^{-\left(\alpha+\sum \beta_{j} X_{j}\right)}\right]}{\left[1+e^{-\left(\alpha+\sum \beta_{j} X_{j}\right)}\right]}-\sum_{i=n_{1}+1}^{N} \frac{X_{j}\left[e^{-\left(\alpha+\sum \beta_{j} X_{j}\right)}\right] \cdot\left[1+e^{+\left(\alpha+\beta_{j} X_{j}\right)}\right]}{\left[1+e^{-\left(\alpha+\sum \beta_{j} X_{j}\right)}\right]^{2}}=0 \\
& j=1, \ldots, k
\end{aligned}
$$

Os parâmetros estimados pelo método da máxima verossimilhança são consistentes, assintoticamente normais e eficientes. Este trabalho utilizou o método da Máxima PseudoVerossimilhança (MPV) ${ }^{4}$ em função de trabalhar com um plano amostral complexo, neste caso em específico a PNAD, onde segundo Binder (1983) incorpora estratificação, conglomeração, probabilidades desiguais de seleção e ajustes de pesos amostrais para calibração com os totais populacionais.

O método de MPV permite estimativas plausíveis e, além do que, é simples de calcular em ambos os casos, ou seja, ao calcular os estimadores e também as variâncias dos estimadores dos parâmetros. O MPV oferece suporte para o do desenvolvimento de rotinas que permitem de incorporar adequadamente os efeitos de planos amostrais complexos a partir de softwares como Stata, SAS, e outros aplicativos (BATTISTI, 2008).

4 Para detalhes da MPV ver Pessoa e Silva (1998) e Silva et al. (2002) 


\subsection{Interpretaçáo dos Coeficientes do Modelo de Regressáo Logística}

Segundo Pindyck e Rubinfeld, (1998), o modelo também pode ser construído através de uma estrutura não linear, em que o modelo logit permite obter razóes de chances, e isto facilita as interpretaçóes dos resultados. Desta forma, pode se verificar a chance do indivíduo idoso estar inserido no mercado de trabalho dado à característica de comparação analisada, neste caso em especifico, os indivíduos idosos que não estão inseridos no mercado de trabalho. A interpretação dos parâmetros estimados neste trabalho é feita por meio do cálculo da razão de chance (odds ratio) e é representada pela seguinte expressão:

$$
p\left(z_{i}\right)=\frac{e^{\left(\alpha+\sum_{j} \beta_{j} x_{i j}\right)}}{1-e^{\left(\alpha+\sum_{j} \beta_{j} x_{i j}\right)}} \rightarrow \frac{p\left(z_{i}\right)}{1-p\left(z_{i}\right)} \rightarrow e^{x \prime \beta} \rightarrow \ln \frac{p\left(z_{i}\right)}{1-p\left(z_{i}\right)}=x^{\prime} \beta
$$

Para esta análise, com relação às variáveis explicativas, a razão de chance estimada demonstra a diferença do evento ocorrer em relaçáo à categoria base. Neste caso, ao ocorrer um valor positivo do coeficiente, a chance de o indivíduo idoso estar inserido no mercado de trabalho é maior do que a categoria base, enquanto um coeficiente negativo indica que essa chance é menor.

É importante analisar a qualidade do ajustamento do modelo que foi estimado. Nesta tarefa, verificam-se de forma associada e de forma individual as variáveis, utiliza-se a base teórica e os testes estatísticos para determinar as variáveis no modelo.

A partir dos níveis de significância dos parâmetros estimados do modelo, é possível a sua avaliação, com base na estatística Wald (WOOLDRIDGE, 2006). Semelhante à estatística $t$ dos mínimos quadrados ordinários, onde se testa a hipótese nula de que cada coeficiente associado às variáveis é estatisticamente diferente de zero (GREENE, 2003).

\subsection{Base de dados e descrição das variáveis}

Os dados utilizados são provenientes da PNAD, fornecida pelo IBGE para os períodos de 2004 e 2014, referentes aos estados brasileiros. Estes dados serão utilizados na formulação do modelo a fim de captar as variáveis que contribuem para que o idoso esteja inserido no mercado de trabalho brasileiro.

A PNAD coleta informaçóes anuais sobre características demográficas e socioeconômicas da população e características dos domicílios. Para o presente estudo apenas os dados referentes a pessoas foram utilizados.

Antes de serem trabalhadas, as variáveis devem passar por alguns critérios e controles. Apenas serão considerados os dados das pessoas com idade igual ou superior a 60 anos. Nos estudos sobre essa parcela da população, Camarano e Medeiros (1999), utilizam essa faixa etária, pois, se enquadra na Política Nacional do Idoso.

Outro parâmetro que deve ser observado, é que apenas idosos do setor urbano serão contemplados no estudo, em vista que, há diferenças nos regimes de aposentadoria entre residentes no meio urbano e rural. Ainda no meio urbano as mulheres podem se aposentar com idade diferenciada dos homens, 55 anos. Dessa forma, a oferta de trabalho entre homens e mulheres pode ser afetada de maneira heterogênea no meio rural ou urbano. Não 
considerar os idosos do setor rural, causa uma melhor observação do efeito da aposentadoria na escolha de continuar ou não trabalhando (LIBERATO, 2003).

Após os critérios citados, haverá a separação das variáveis, divididas em categorias pessoais, relativas ao sexo, idade, raça. Também serão separados pelo grau de instrução (educação), relativos aos anos de estudos dos idosos da amostra, podem ser: estudoO (que não estudaram), estudo1a (entre 1 e 4 anos de estudo), estudo5a10 (entre 5 e 10 anos de estudo), estudo1 1a14 (entre 11 e 14 anos de estudo), estudo15 (15 anos de estudo ou mais). A condição de aposentando e o logaritmo da renda também foram inseridos no estudo.

A situaçâo nas famílias também será contemplada na pesquisa, quantas pessoas residem no domicílio, se ele é o responsável pela família e se tem casa própria. Seguindo, as categorias serão divididas pelo setor de atividade, tais como: setor agrícola ou agropecuário, setor industrial, setor de construção civil, setor de comércio, setor de educação, saúde e serviços sociais; ainda sobre as características do emprego, se o idoso trabalha com carteira assinada, também é inserido no estudo.

Após ser realizada a limpeza dos dados, excluindo-se os dados missing, a amostra foi composta por 7.088 observaçôes no ano de 2004 e 9.516 observações para o ano de 2014 . A Tabela 1, a seguir, apresenta a descrição da amostra selecionada para análise empírica. A primeira coluna traz as estatísticas para o ano de 2004 e a segunda para o ano de 2014, considerando os dados da PNAD.

Tabela 1 - Estatística Descritiva das variáveis para os anos de 2004 e 2014

\begin{tabular}{l|c|c|c|c}
\hline \multirow{2}{*}{ Variável } & \multicolumn{2}{c|}{$\mathbf{2 0 0 4}$} & \multicolumn{2}{c}{$\mathbf{2 0 1 4}$} \\
\cline { 2 - 5 } Gênero & Média & Desvio-padrão & Média & Desvio-padrão \\
\hline Mulher* & & & & \\
\hline Homem & 0,357487 & 0,47929 & 0,374843 & 0,494342 \\
\hline Raça & 0,642513 & 0,47929 & 0,625157 & 0,494342 \\
\hline Não Branco* & & & & \\
\hline Branco & 0,468147 & 0,499016 & 0,516788 & 0,499742 \\
\hline Faixa etária & 0,531853 & 0,499016 & 0,483212 & 0,499742 \\
\hline idade 60 a 69 & & & & \\
\hline idade 70 a 79 & 0,646827 & 0,477986 & 0,516594 & 0,499749 \\
\hline idade 80 mais* & 0,316498 & 0,465139 & 0,442284 & 0,496682 \\
\hline Renda & 0,025 & 0,156135 & 0,032221 & 0,176594 \\
\hline lnrenda & & & & \\
\hline Aposentado & 5,913215 & 1,018126 & 7,020547 & 0,848543 \\
\hline Näo* & & & & \\
\hline Sim & 0,530076 & 0,499126 & 0,466667 & 0,498912 \\
\hline C_Carteira & 0,469924 & 0,499126 & 0,533333 & 0,498912 \\
\hline Não* & & & & \\
\hline Sim & 0,893373 & 0,308661 & 0,83756 & 0,368873 \\
\hline & 0,106627 & 0,308661 & 0,16244 & 0,368873 \\
\hline
\end{tabular}




\begin{tabular}{|c|c|c|c|c|}
\hline \multirow[b]{2}{*}{ Variável } & \multicolumn{2}{|c|}{2004} & \multicolumn{2}{|c|}{2014} \\
\hline & Média & Desvio-padrão & Média & Desvio-padrão \\
\hline \multicolumn{5}{|l|}{ Escolaridade } \\
\hline $\begin{array}{l}\text { estudo } 0 \\
\text { estudo } 1 \text { a } 4\end{array}$ & $\begin{array}{l}0,34854 \\
0,35501 \\
\end{array}$ & $\begin{array}{l}0,476548 \\
0,478556\end{array}$ & $\begin{array}{l}0,176972 \\
0,311756 \\
\end{array}$ & $\begin{array}{l}0,381663 \\
0,463233\end{array}$ \\
\hline estudo 5 a 10 & 0,197578 & 0,398205 & 0,27044 & 0,444209 \\
\hline estudo 11 a 14 & 0,084937 & 0,278811 & 0,173585 & 0,378771 \\
\hline estudo 15 mais & 0,084771 & 0,278564 & 0,115723 & 0,319908 \\
\hline \multicolumn{5}{|c|}{ Chefe de domicílio } \\
\hline Não* & 0,177339 & 0,381987 & 0,277213 & 0,447644 \\
\hline Sim & 0,822661 & 0,381987 & 0,722787 & 0,447644 \\
\hline \multicolumn{5}{|l|}{ Setor do emprego } \\
\hline Agrícola & 0,258092 & 0,437615 & 0,158353 & 0,36509 \\
\hline Indústria & 0,1219 & 0,327193 & 0,118948 & 0,323744 \\
\hline Comércio & 0,234272 & 0,423573 & 0,239363 & 0,426717 \\
\hline Const Civil & 0,069917 & 0,255026 & 0,105429 & 0,307121 \\
\hline Educ_sau_soc & 0,063052 & 0,243073 & 0,079753 & 0,270924 \\
\hline Outros & 0,252767 & 0,434629 & 0,298156 & 0,457472 \\
\hline \multicolumn{5}{|l|}{ Moradia } \\
\hline Própria & 0,866218 & 0,34044 & 0,857794 & 0,349279 \\
\hline Não & 0,133782 & 0,34044 & 0,142206 & 0,349279 \\
\hline Tam. da família & 3,398985 & 1,957785 & 2,864248 & 1,501388 \\
\hline Observaçóes & \multicolumn{2}{|c|}{7088} & \multicolumn{2}{|c|}{9516} \\
\hline
\end{tabular}

Fonte: Elaborado pelos autores com base na PNAD de 2004 e 2014.

Obs.: * Categoria de referência em variável qualitativa. Para variáveis qualitativas a média equivale à proporção.

Os dados mostram que, para o ano de 2004, em média, 64\% dos idosos que estavam trabalhando eram homens, contra 35\% de mulheres idosas. Para o ano de 2014, a média dos idosos que estavam trabalhando era de $62 \%$ de homens, contra $37 \%$ de mulheres. De acordo com a raça, no ano de 2004 a parcela de idosos que estava inserida no mercado de trabalho era de 53\% de brancos, no ano de 2014 essa parcela caiu para 48\% dos idosos. Em relação a ser chefe da família, em 2004 essa parcela era em média de 82\% dos idosos e também teve queda em comparação a 2014 que passou para $72 \%$ do grupo analisado. Trabalhos com carteira assinada em 2004 representaram em média 10\% dos idosos inseridos no mercado de trabalho, em 2014 esse número passou para $16 \%$.

Ao considerar os grupos etários, para o ano de 2004, os idosos entre 60 e 69 anos são os mais ativos no mercado de trabalho, cerca de $64 \%$ contra $31 \%$ dos idosos entre 70 e 79 anos e apenas $2 \%$ contra os idosos entre 80 anos ou mais. Para o ano de 2014, os idosos entre 60 e 69 anos também são os mais ativos no mercado de trabalho, cerca de $51 \%$ contra $44 \%$ dos idosos entre 70 e 79 anos, e apenas 3\% contra os idosos entre 80 anos ou mais.

Em relação à escolaridade, no ano de 2004, os idosos que possuem ensino fundamental 1 (de 1 a 4 anos de estudos) são a maioria no mercado de trabalho, cerca de 
$35 \%$, enquanto que sem instrução representam 34\% dos trabalhadores, seguidos de $19 \%$ do nível fundamental 2 (de 5 a 10 anos de estudo), $8 \%$ de idosos com ensino médio (entre 11 e 14 anos de estudos) e $8 \%$ com ensino superior (15 anos ou mais de estudos). Para o ano de 2014, os idosos que possuem ensino fundamental 1 (de 1 a 4 anos de estudos) são a maioria no mercado de trabalho, cerca de $31 \%$, seguidos de $27 \%$ do nível fundamental 2 (de 5 a 10 anos de estudo), enquanto que ensino médio (entre 11 e 14 anos de estudos) representam $17 \%$ dos trabalhadores, $7 \%$ de idosos com ensino médio (entre 11 e 14 anos de estudos), $11 \%$ com ensino superior ( 15 anos ou mais de estudos) e idosos sem instrução representam $17 \%$ no mercado de trabalho. Os que se encontram na condição de chefe da família representam $82 \%$ dos idosos no ano de 2004 e no ano de 2014 são representados por $72 \%$ deste grupo analisado.

A parcela de idosos aposentados no ano de 2004 era de $46 \%$, contra $53 \%$ de náo aposentados. Já no ano de 2014, a parcela de idosos aposentados era de aproximadamente $53 \%$, contra $46 \%$ de idosos náo aposentados. No ano de 2004 , os setores de atividades que mais empregaram idosos foram os setores agrícola com $25 \%$, seguido de outras atividades comércio com $25 \%$, o setor de comércio representou $23 \%$, o setor de indústria representou $12 \%$ da força de trabalho idosa, a construçâo civil $6 \%$, e por fim, Educaçáo saúde e serviços sociais $6 \%$. No ano de 2014 , o setor de outras atividades que concentrou a maior parcela de mão de obra idosa com $27 \%$, seguido do setor do comércio que registrou $23 \%$, o setor agrícola $15 \%$, a indústria 11 a construção civil 10\%, e por fim, Educação saúde e serviços sociais $7 \%$.

No ano de 2004, uma parcela de $86 \%$ dos idosos possuía casa própria. No ano de 2014 , cerca de $85 \%$ dos idosos possuíam casa própria. A renda dos domicílios dos idosos em 2004 era em média de 5,9 salários mínimos e em 2014 era em média de 7,02 salários mínimos por família. E por fim, o tamanho das famílias dos idosos em 2004 era de 3,39 pessoas e em 2014 era de 2,86 membros.

\section{RESULTADOS}

Nesta seçáo, são apresentados os resultados obtidos por meio da estimação do modelo logit para os anos de 2004 e 2014, a partir das respectivas PNADs. Os resultados apresentados do modelo microeconométrico estão demonstrados em razōes de chances (odds ratio $)^{5}$. Serão também apresentados posteriormente, os cenários elaborados de inserçâo no mercado de trabalho para o ano de 2014.

\subsection{Resultados do modelo logit (Odds Ratio)}

A partir do banco de dados elaborado de acordo com a PNAD de cada ano analisado (2004 e 2014), foram elaboradas duas regressóes as quais podem ser observados seus respectivos resultados na Tabela 2 , a seguir.

5 Para maiores informações com relação a leitura dos resultados em Odds Ratio ver na subseção 3.2. 
Tabela 2 - Razóes de chances para os Idosos inseridos no mercado de trabalho - 2004 e 2014 (continua)

\begin{tabular}{|c|c|c|}
\hline \multirow{2}{*}{ Variáveis } & \multicolumn{2}{|c|}{ Logit $(O R)$} \\
\hline & 2004 & 2014 \\
\hline \multirow[t]{2}{*}{ Masculino } & $3.714^{* * *}$ & $2.970^{* * *}$ \\
\hline & $(0.436)$ & $(0.308)$ \\
\hline \multirow[t]{2}{*}{ Chefefamilia } & 0.870 & 0.966 \\
\hline & $(0.105)$ & $(0.0961)$ \\
\hline \multirow[t]{2}{*}{ Branca } & $0.748^{* * *}$ & $0.818^{* *}$ \\
\hline & $(0.0830)$ & $(0.0781)$ \\
\hline \multirow[t]{2}{*}{ idade60a69 } & 0.891 & 1.151 \\
\hline & $(0.288)$ & $(0.297)$ \\
\hline \multirow[t]{2}{*}{ idade70a79 } & 0.814 & 1.320 \\
\hline & $(0.267)$ & $(0.334)$ \\
\hline \multirow[t]{2}{*}{ lnrenda } & $1.385^{* * *}$ & $1.380^{* * *}$ \\
\hline & $(0.100)$ & $(0.101)$ \\
\hline \multirow[t]{2}{*}{ estudoO } & 1.266 & 0.971 \\
\hline & $(0.262)$ & $(0.195)$ \\
\hline \multirow[t]{2}{*}{ estudo1a4 } & 1.003 & 0.905 \\
\hline & $(0.173)$ & $(0.151)$ \\
\hline \multirow[t]{2}{*}{ estudo5a10 } & 0.921 & 0.916 \\
\hline & $(0.128)$ & $(0.142)$ \\
\hline \multirow[t]{2}{*}{ estudo11a14 } & 0.914 & 0.842 \\
\hline & $(0.230)$ & $(0.161)$ \\
\hline \multirow[t]{2}{*}{ aposentado } & $1.357^{* * *}$ & $1.320^{* * *}$ \\
\hline & $(0.142)$ & $(0.130)$ \\
\hline \multirow[t]{2}{*}{ C_carteira } & $0.541^{* * *}$ & $0.550^{* * *}$ \\
\hline & $(0.115)$ & $(0.0919)$ \\
\hline \multirow[t]{2}{*}{ agricola } & $0.0469^{* * *}$ & $0.0363^{* * *}$ \\
\hline & $(0.00875)$ & $(0.00546)$ \\
\hline industria & $2.027^{* *}$ & 1.249 \\
\hline \multirow[t]{2}{*}{ construçâocivil } & $\begin{array}{c}(0.562) \\
0.164^{* * *}\end{array}$ & $\begin{array}{c}(0.300) \\
0.203^{* * *} \\
\end{array}$ \\
\hline & $(0.0430)$ & $(0.0416)$ \\
\hline \multirow[t]{2}{*}{ comercio } & $2.322^{* * *}$ & $1.961^{* * *}$ \\
\hline & $(0.643)$ & $(0.439)$ \\
\hline \multirow[t]{2}{*}{ Educ_sau_soc } & 0.980 & 1.085 \\
\hline & $(0.299)$ & $(0.281)$ \\
\hline \multirow[t]{2}{*}{ CasaProp } & $0.664^{* * *}$ & 0.910 \\
\hline & $(0.102)$ & $(0.143)$ \\
\hline
\end{tabular}




\begin{tabular}{l|c|c}
\hline \multirow{2}{*}{ Variáveis } & \multicolumn{2}{|c}{ Logit $(O R)$} \\
\cline { 2 - 3 } & 2004 & 2014 \\
\hline$N \_c o m p o n$ & $1.093^{* * *}$ & $1.122^{* * *}$ \\
\hline Constante & $(0.0349)$ & $(0.0424)$ \\
\hline & 2.981 & 1.558 \\
\hline Observaçóes & $(1.981)$ & $(1.094)$ \\
\hline
\end{tabular}

Obs.: *Erro padrão em parênteses. Níveis de significâncias: ${ }^{* * *} \mathrm{p}<0.01,{ }^{* *} \mathrm{p}<0.05,{ }^{*} \mathrm{p}<0.1$.

Fonte: Elaborado pelos autores com base nos dados das Pnad de 204 e 2014.

Para verificar se o modelo possui um bom ajustamento, foram feitos testes utilizando a estatística de Wald. Verificou que as variáveis utilizadas são conjuntamente significativas, com probabilidade caudal de nulidade conjunta dos parâmetros igual a $0 \%$ para todos os modelos.

Os resultados demonstram que a maioria das variáveis selecionadas são estatisticamente significativas para explicar as chances de o indivíduo ser idoso e estar inserido no mercado de trabalho.

As variáveis referentes às características pessoais, como sexo, demonstram que os homens idosos apresentam chances positivas de estarem inseridos no mercado de trabalho de aproximadamente $271 \%$ em relação às mulheres, para o ano de 2004 . No ano de 2014 , essa razão de chances é de $197 \%$. Em relação à idade dos idosos, para o ano de 2004, ter entre 60 e 69 anos, diminui as chances de trabalhar em $11 \%$ e ter entre 70 e 79 as chances de estar inserido no mercado de trabalho diminuem em 19\% em relação aos idosos de 80 anos ou mais. Para o ano de 2014, ter entre 60 e 69 anos aumenta a chance de trabalhar em $15 \%$ e entre 70 e 79 anos o aumento é ainda maior, a chance de trabalhar é de $32 \%$ em relação aos idosos com 80 anos ou mais. Esses resultados estáo de acordo com as evidencias de Camarano (2001), no qual destaca a importância da sexualidade e idade dos idosos na chance dos idosos estarem inseridos no mercado de trabalho.

Em relaçáo à raça do indivíduo, demonstra que os brancos têm respectivamente, chances negativas de 26\% no ano de 2004 e chances negativa de 19\% no ano de 2014 dos idosos estarem inseridos no mercado de trabalho em relaçáo aos não brancos. Este resultado está de acordo com Moura e Cunha (2010) onde fica evidente que a populaçáo branca tem menor chance de estar no mercado de trabalho.

A escolaridade apresenta uma relação negativa com a inserção do idoso no mercado de trabalho nos anos de 2004 e de 2014 em relaçáo a quem tem 15 anos de estudo ou mais, sugerindo que quanto maior o grau de instrução do idoso, maior é a chance de se aposentar. Esses resultados estão de acordo com a pesquisa realizada por Queiroz e Ramalho (2005), tanto em relação a não significância das variáveis no modelo quanto à análise realizada das mesmas.

As variáveis referentes às características do núcleo familiar do trabalhador, a condição de ser o chefe da família, ou seja, o maior responsável pelo sustento da mesma apresentou na estimação um efeito negativo na condição de ofertar trabalho nos dois anos estudados. 
Se o trabalhador for o chefe da família apresentará uma razão de chance em $13 \%$ menor de disponibilizar mão de obra no ano de 2004 e de aproximadamente $4 \%$ menor de disponibilizar mão de obra no ano de 2014. Esses resultados não estáo de acordo com a literatura encontrada sobre o tema.

Em relação ao tamanho da família, os dados de 2004 mostram que, a cada pessoa que for adicionada, as chances de o indivíduo idoso trabalhar aumentam em 9\%. Já no ano de 2014, cada membro adicional na unidade familiar apresenta chance positiva de aproximadamente $12 \%$ de o idoso ofertar trabalho. Esse resultado está em concordância com Camarano (2001), onde os idosos participavam com mais da metade da renda familiar.

Os setores que apresentam maiores chances do idoso ofertar trabalho no ano de 2004 são: comercio com 132\%, na indústria com 102\%. Os outros setores apresentam chance negativa: o setor agrícola em $96 \%$, o setor da construção civil em $84 \%$ e educação, saúde e serviços sociais em $2 \%$. No ano de 2014, os setores que apresentaram maior chance do idoso estar inserido no mercado de trabalho foram: o comercio com $96 \%$, a indústria com $25 \%$, educação, saúde e serviços sociais com $8 \%$. Os outros setores apresentaram chances negativas: o setor agrícola em $97 \%$ e a construçâo civil em $80 \%$. Esses resultados corroboram com o trabalho realizado por Queiroz (2014) onde destacou quais atividades são ocupadas pelos idosos.

A condição de aposentado apresenta para o ano de 2004 um resultado positivo em $35 \%$ e nos anos de 2014 positivos em 32\%. Esses resultados não estão de acordo com a literatura, onde a condição de aposentado apresenta chances negativas de o idoso estar inserido no mercado de trabalho. Porém esse resultado indica a necessidade do idoso completar a sua renda a fim de garantir a continuidade ou a melhora do seu padrão de vida.

Em relação aos trabalhos com carteira, no ano de 2004, estar com a carteira assinada diminui em $46 \%$ a chance de o idoso estar inserido no mercado de trabalho, valor que quase não se alterou no ano de 2014, que foi de 45\%. De acordo com Queiroz (2009), os empregos sem carteira assinada ou autônomos servem como alternativa de trabalho para idosos com elevado nível de estudo.

Os resultados também sugerem que o fato do idoso ter casa própria diminui as chances de ele continuar inserido no mercado de trabalho, no ano de 2004 diminuiu em $34 \%$ e no ano de 2014 em 9\%. Essa variável mostra que, como quando o idoso não precisa pagar aluguel ele está menos propenso a ofertar mão de obra. Variável essa que não foi encontrada na literatura sobre o assunto.

Por fim, com relação aos efeitos apresentados pela variável ln da renda familiar, constata-se que a elevação da renda provoca aumento nas chances de emprego. Um aumento de $1 \%$ na renda familiar aumenta a chance de que o idoso seja ativo em $38 \%$ em ambos os anos. Estes resultados estão de acordo com Pérez; Wajnman e Oliveira (2006), que destacam que a renda familiar pode indicar o nível de consumo mais elevado, o que levaria o indivíduo a continuar trabalhando para manter esse nível. 


\subsection{Cenários de inserção do idoso no mercado de trabalho.}

Com base nos resultados obtidos, foi de conveniência analisar cenários de inserção no mercado de trabalho para o ano de 2014, elaborados e estimados com estrutura de modelo logit, a fim de mostrar como se comportam as chances dos idosos estarem inseridos no mercado de trabalho, dado as características pré-fixadas.

Num primeiro cenário, gráfico 1, os idosos foram separados pelo sexo (masculino e feminino) e na condição de aposentados e não aposentados. A partir desses parâmetros fixos, foram inseridos anos de estudos para verificar quais alteraçóes possíveis na condição de ofertar trabalho por parte do idoso. Tanto o idoso quanto a idosa aposentadas não alteraram a sua predisposição quanto à oferta de trabalho. Já os idosos não aposentados, tanto os homens quanto as mulheres aumentaram em $1 \%$ as chances de ofertar trabalho.

Gráfico 1 - Probabilidades dos idosos estarem no mercado de trabalho de acordo com a aposentadoria e a escolaridade

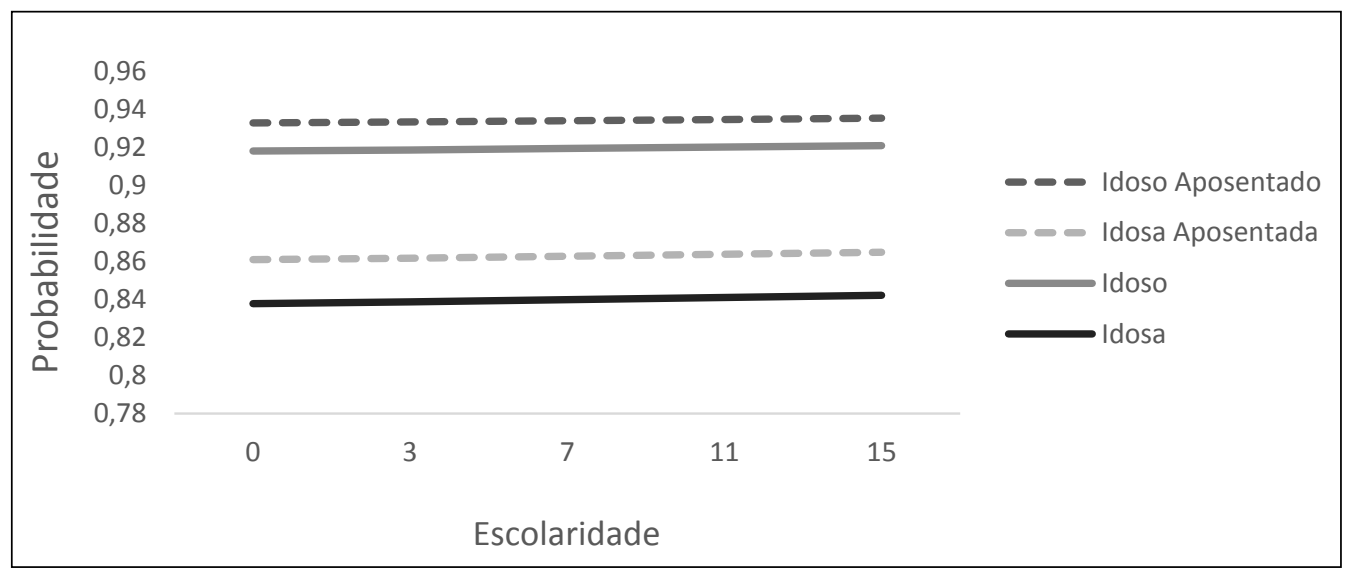

Fonte: Elaborado pelos autores a partir das informaçôes da PNAD 2014.

No segundo cenário, gráfico 2, os idosos foram separados por sexo (masculino e feminino) e na condição de estar aposentada e com carteira assinada, onde o fator variável é a renda desse idoso em salários mínimos. Todos os resultados apresentaram aumento nas chances de estar inserido no mercado de trabalho: o idoso homem, aposentado e com carteira aumentou de $81 \%$ para $92 \%$; a idosa mulher aposentada e com carteira aumentou de $68 \%$ para $87 \%$ de chance; o idoso com carteira passou de $78 \%$ para $92 \%$ e a idosa com carteira passou de $63 \%$ para $85 \%$. 
Gráfico 2 Probabilidade dos idosos estarem no mercado de trabalho de acordo com aposentadoria, carteira assinada e faixas de renda

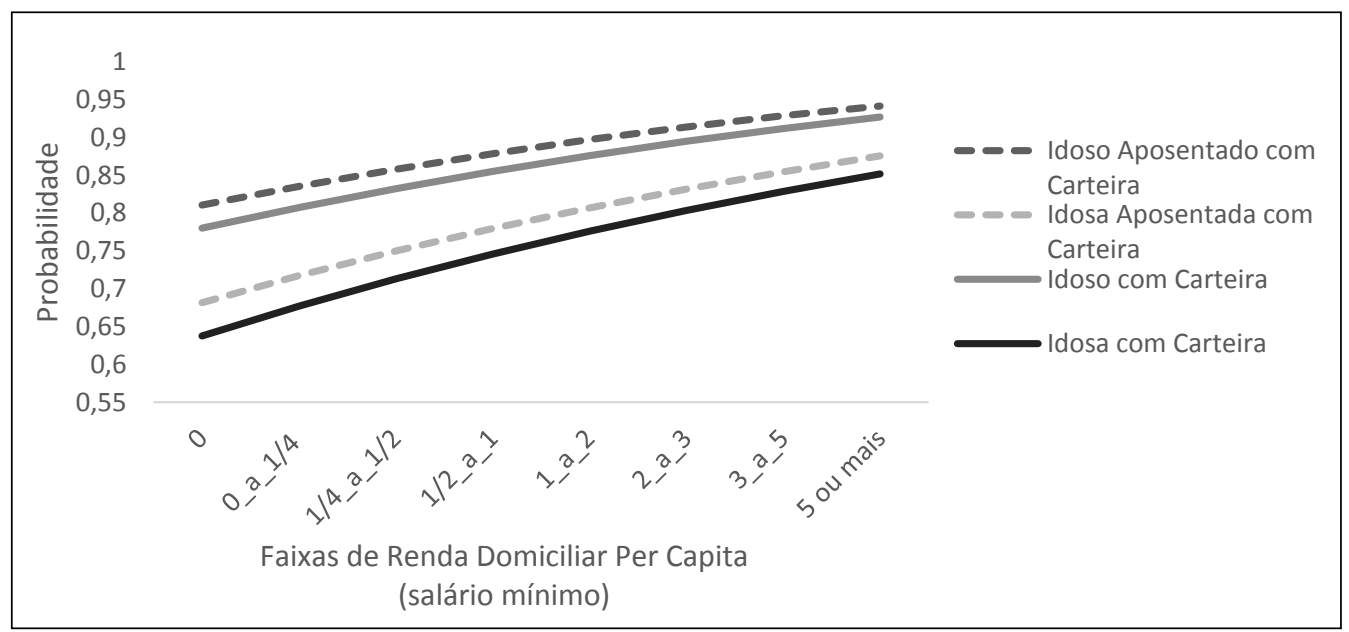

Fonte: Elaborado pelos autores a partir das informaçôes da PNAD 2014.

A partir dos resultados, é possível notar que quanto mais elevada é a renda do idoso, maior é a sua necessidade de continuar inserido no mercado de trabalho, justamente para manter seu padrão de vida. Assim como, é notável a diferença em relação aos homens e mulheres na inserção no mercado de trabalho, corroborando com os resultados do presente trabalho assim como a literatura pertinente.

\section{CONSIDERAÇÓES FINAIS}

Este trabalho busca avaliar o perfil do trabalhador idoso, em atividade no Brasil nos períodos de 2004 e 2014. Para a realização deste objetivo foi utilizada a base de dados das PNADs de 2004 e 2014.

O modelo empírico utilizado para a formulação deste perfil baseou-se no trabalho nos trabalhos realizados sobre o tema, que utilizaram a metodologia de variáveis dependentes binárias com o modelo logit.

$\mathrm{Na}$ metodologia foi utilizada uma proxy de trabalho, caracterizado aqui por pessoas idosas, considerando idoso idade a partir de 60 anos, tem-se dois eventos possíveis: o primeiro onde o idoso está inserido no mercado de trabalho e a variável assume valor 1 e, o segundo, onde não está inserido no mercado de trabalho e ela assume valor 0 .

As variáveis foram selecionadas com base na teoria microeconômica utilizada pela economia do trabalho e na literatura sobre idosos no mercado de trabalho, e foram tratadas para que o perfil pudesse ser constituído através da comparação entre categorias de referência.

O perfil do trabalhador idoso obtido pelos resultados do modelo logit demonstrou características interessantes no perfil dos idosos inseridos no mercado de trabalho no decorrer de uma década. É de homens idosos a maior possibilidade de trabalhar, não brancos, aposentados e inseridos principalmente no comércio. A principal mudança é em 
relação às faixas de idade, que no ano de 2004 tinham chances negativas de o idoso estarem inseridos no mercado de trabalho, já no ano de 2014 as faixas analisadas apresentaram chances positivas. Indicando que os idosos estáo realmente recorrendo ao mercado de trabalho para completar sua renda.

Os resultados obtidos estão de acordo com a literatura sobre a tendência da mão de obra idosa no país, que indica uma população cada vez mais idosa, ou seja, o aumento da expectativa de vida. O presente estudo apresenta algumas limitaçóes no que se refere que a análise foi realizada de forma estática ao comparar dois anos e não a evoluçáo ao decorrer dos anos através de forma contínua. E a principal limitação é a indisponibilidade de variáveis macroeconômicas na base de dados, que podem interferir na inserção dos idosos no mercado de trabalho.

E dessa forma este trabalho buscou contribuir com esse tema tăo importante para economia, assim como surge à necessidade de estudos mais aprofundado buscando identificar se houve ou náo mudanças na probabilidade do idoso estar inserido no mercado de trabalho, na última década, assim como, o que contribuiu para tal mudança, de forma a compreender melhor, quais fatores ou variáveis que contribuíram mais (ou menos) para os idosos se inserirem no mercado de trabalho.

\section{REFERÊNCIAS}

BATTISTI, I. D. E. Análise de dados epidemiológicos incorporando planos amostrais complexos. Tese. Porto Alegre: UFRGS, 2008.

BEEN, J.; KNOEF, M. The necessity of self-employment towards retirement: evidence from labor market dynamics and search requirements in unemployment insurance. Instituut Gak and Netspar, 2013.

BENÍTEZ-SILVA, H.; HEILAND, F. Early claiming of social security benefits and labor supply behavior of older Americans. Applied Economics. Florida. 40(23): p. 2969-2985, 2008.

BÓS, A. M.; BÓS, A. J. G. RBCEH. A participação dos idosos gaúchos no mercado de trabalho e a força da relação renda-saúde. Revista Brasileira de Ciências do

Envelhecimento Humano. Passo Fundo, v. 1, n.1, p. 48-56, 2004.

CAMARANO, A. A.; MEDEIROS, M.. Introdução. In: CAMARANO, Ana. Amélia. IPEA. Muito Além dos 60? Os Novos Idosos Brasileiros. Rio de Janeiro. p. 1-18, 1999.

CAMARANO, A. A.. O idoso brasileiro no mercado de trabalho. IPEA. Rio de Janeiro. Texto para discussão 830, 2001.

CAMARANO, A. A.. Envelhecimento da populaçáo brasileira: Uma contribuição demográfica. IPEA. Rio de Janeiro. Texto para discussão 858, 2002. 
CAMARANO, A. A.; KANSO, S.; MELLO, J. L. Quão além dos 60 poderão viver os Idosos Brasileiros? In: CAMARANO, A.A (Org.): Os novos idosos brasileiros: muito além dos 60? Rio de Janeiro: IPEA, 2004.

CARRERA-FERNANDEZ, J. ; MENEZES, W.. O idoso no mercado de trabalho: uma análise a partir da Região Metropolitana de Salvador. Revista Econômica do Nordeste. Fortaleza, v. 32, n.1, p. 52-67, 2001.

FRANÇA, L.. Envelhecimento dos trabalhadores nas organizaçóes: estamos preparados? In: L. FRANÇA \& D. STEPANSKY (Orgs.). Propostas multidisciplinares para o bemestar na aposentadoria (p. 25-52). Rio de Janeiro: Quarter/FAPERJ. 2012.

FRANÇA, L. H. de F. P. et al. Aposentar-se ou continuar trabalhando? o que influencia essa decisão? Psicol. cienc. prof., Brasília, v. 33, n. 3, 2013.

FUCHS, V. R. Self-employment and labor force participation of older males. Journal of Human Resources, v. XVII, n. 3, p. 339-357, 1982.

FUNDAÇÃO PERSEU ABRAMO. Idosos no Brasil vivências, desafios e expectativas na 3 idade. Disponível em: <http://www2.fpa.org.br/portal/modules/wfdownloads/ viewcat.php?cid=69>. Acesso em 12 Jan. 2015.

FURTADO. A.. A participaçáo do Idoso no Mercado de Trabalho Brasileiro.

Consultoria Legislativa da Câmara dos Deputados, 2005. Disponível em: <http://www2. camara.gov.br/publicacoes/estnottec/tema8/2004_13576.pdf>. Acesso em: 20 jan. 2015.

GIATTI, L.; BARRETO, S. M.. Saúde, trabalho e envelhecimento no Brasil. Cad. Saúde Pública. 2003, vol.19, n.3, pp. 759-771.

GASPARINI, L. et al.. World Economic and Social Survey. Poverty among the elderly in Latin America and the Caribbean. Universidad Nacional de La Plata. Argentina, 2007.

GREENE, W. H.. Econometric analysis. 5. ed. New Jersey: Prentice Hall, 2003.

GRUBER, J.; WISE, D.. Social security and retirement: An international comparison. American Economics Review. EUA. 88(2): p.158-163, 1998

HECKMAN, J.. Sample selection bias as a specification error. Econometrica. Issue. 47:153-162, 1979.

INSTITUTO BRASILEIRO DE GEOGRAFIA E ESTATÍSTICA. Perfil dos Idosos

Responsáveis pelos domicílios. Disponível em: <http://www.ibge.gov.br/home/ presidencia/noticias/25072002pidoso.shtm>. Acesso em: 15 de Out. 2014. 
LEESON, G. W.; HARPER, S. Some descriptive findings from the Global Ageing Survey. Oxford Institute of Ageing, University of Oxford, Research Report, Oxford. v. 108, 2008.

LEME, M. C. S.; MÁLAGA, T.. Entrada e saída precoce da força de trabalho: Incentivos do regime de previdência brasileiro. Revista Brasileira de Economia. Rio de Janeiro. 55:205-222, 2001.

LIBERATO, V. C.. A oferta de trabalho masculina “pós-aposentadoria” Brasil urbano - 1981/2001. 2003. 78 f. Dissertação (Mestrado em economia) Faculdade de Ciências Econômicas de Minas Gerais (UFMG), Belo Horizonte. 2003.

MINISTÉRIO DA PREVIDÊNCIA SOCIAL. Projeçóes atuariais para o regime geral de previdência social - RGPS. Secretaria de Políticas de Previdência Social. Brasília (DF), abril, 2011.

MOURA, C. S.; CUNHA, M. S.. Fatores determinantes da participação e do rendimento do idoso no mercado de trabalho. A Economia em Revista. Maringá. v. 18 No 2, 2010.

NASIR, Z. M.. Analysis of occupational choice in Pakistan: A multinomial approach. The Pakistan Development Review. 44(1):57-79, 2005.

NORMANHA FILHO, M. A.. A permanência ou reinserção do idoso no mercado de trabalho: uma alternativa para comunidades voltadas ao desenvolvimento sustentável e à valorização da cultura local. Revista Gerenciais. v. 3, p. 79-86. São Paulo: UNINOVE, out. 2004.

OLIVEIRA, F. E. B., BELTRÃO, K. I., \& FERREIRA, M. G.. Reforma da Previdência. Rio de Janeiro: IPEA, Texto para Discussão 508, 1997.

ORGANIZAÇÃO DAS NAÇÓES UNIDAS. World. Population Ageing 2009. New York. Disponível em: <http://www.un.org/esa/population/publications/WPA2009/ WPA2009_WorkingPaper.pdf>. Acessado em: 12 dezembro 2014.

PÉREZ, E. R.; WAJNMAN, S. ; OLIVEIRA, A. M. H. C. de . Análise dos Determinantes da Participação no Mercado de Trabalho dos Idosos em Sáo Paulo. Revista Brasileira de Estudos de População. São Paulo. v. 23, p. 269-286, 2006.

PINDYCK, R. S.; RUBINFELD, D. L. Econometric models and economic forecasts. $4^{\circ}$ ed. McGraw-Hill, 1998.

QUEIROZ, V. S.; RAMALHO, H. M. B.. A escolha ocupacional dos idosos no mercado de trabalho: Evidências para o Brasil. Revista EconomiA Selecta. Brasília. v.10, n.4, p.817-848, 2009. 
QUEIROZ, V. S.; RAMALHO, H. M. B.; CAVALCANTI, G. de A.. O Emprego do Idoso no Mercado de Trabalho: Evidências para o Brasil a Partir da PNAD de 2005. In: Anais do XIII Encontro Regional de Economia, Fortaleza. ANPEC, 2005.

SAAD, P. M.. In: CAMARANO, A. A.. (Org.). IPEA. Muito Além dos 60? Os Novos Idosos Brasileiros. Rio de Janeiro.1999, p.251-280.

TAYLOR, M. P. Earnings, independence or unemployment: Why become self-employed? Oxford Bulletin of Economics and Statistics, Oxford. v. 58, n. 2, p. 253-266, 1996.

TEIXEIRA, G. da S.. A padronizaçáo do programa de seguro-desemprego brasileiro promove a equidade? Uma discussão de acordo com o modelo principal-agente com seleção adversa. 2009. 70f. Dissertaçáo (Mestrado em economia) - Pós-Graduação em Economia, Universidade Federal da Paraíba (UFPB), João Pessoa, 2009.

WAJNMAN, S.; OLIVEIRA, A. M. H. C.; OLIVEIRA. E. L.. In: CAMARANO, Ana Amélia. (Org). IPEA. Os Novos Idosos Brasileiros Muito Além dos 60? Rio de Janeiro. P. $453-480,2004$

WAJNMAN, S.; OLIVEIRA, A. M. H. C.; OLIVEIRA. E. L.. In: CAMARANO, Ana Amélia. (org). IPEA. Muito além dos 60? os novos idosos brasileiros. Rio de Janeiro. p. 181-220, 1999.

WOOLDRIDGE, J. M. Introduçáo à econometria: uma abordagem moderna. Pioneira Thomson Learning, 2006. 